\title{
Composição corporal funcional: breve revisão
}

\author{
Functional body composition: a brief review
}

DOI: http://dx.doi.org/10.36453/2318-5104.2018.v16.n1.p235

\section{Anderson dos Santos Carvalho ${ }^{1,3}$, Thiago Cândido Alves ${ }^{1,3}$, Pedro Pugliesi Abdalla², Ana Claudia Rossini Venturini ${ }^{2,3}$, Pablo Dogomar Llanes Leites ${ }^{3}$, Dalmo Roberto Lopes Machado 1,2,3}

\author{
${ }^{1}$ Escola de Enfermagem de Ribeirão Preto da Universidade de São Paulo (EERP-USP) \\ ${ }^{2}$ Escola de Educação Física e Esporte de Ribeirão Preto da Universidade de São Paulo (EEFERP-USP) \\ ${ }^{3}$ Grupo de Estudo e Pesquisa em Antropometria, Treinamento e Esporte (GEPEATE)
}

\section{RESUMO}

Compreender a Composição Corporal Funcional (CCF) é fundamental para que os profissionais que trabalham com o corpo e o movimento possam entender as alterações que ocorrem no organismo. De um ponto de vista histórico, a análise da Composição Corporal (CC) era organizada em dois compartimentos, sendo eles Massa Gorda (MG) e Massa Isenta de Gordura (MIG). A CCF associa componentes da CC a vias metabólicas, fisiológicas e bioquímicas, verificando a influência do movimento e proporcionando ao profissional o entendimento básico da CC através dos diferentes modelos de níveis compartimentais e métodos, com vistas a um entendimento mais efetivo por parte deste. Estudos têm evidenciado a relação entre CC desejável e a saúde dos indivíduos. Portanto, o objetivo foi realizar uma breve revisão da literatura sobre CCF e compreender sua influência no organismo, articulada ou não à prática de atividade física (AF). O presente estudo foi realizado a partir de uma revisão narrativa acerca do tema CCF. Como conclusão, a CC é capaz de influenciar o funcionamento do corpo humano e, atualmente, pode ser analisada em cinco níveis. Quanto mais sofisticada a sua quantificação, melhor é o entendimento sobre sua relação com a variação da condição de saúde do organismo (estado nutricional, metabolismo e patologias). O exercício pode beneficiar a CC, mantendo e aumentando os componentes que influenciam positivamente a saúde.

PALAVRAS-CHAVE: Composição Corporal; Movimento; Saúde.

\section{ABSTRACT}

Understanding Functional Body Composition (FBC) is essential so that professionals working with the body and movement can understand the changes that occur in the body. From a historical point of view, the body composition analysis (BC) was organized into two compartments, being fat mass (FM) and fat-free mass (FFM). The FBC associates BC components to metabolic, physiological and biochemical verifying the movement influence and allowing the professional to have a basic understanding of the $\mathrm{BC}$ through the different compartmental levels models and methods, seeking a more effective understanding. Studies have shown a relationship between a desirable $\mathrm{BC}$ and health of individuals. Therefore, the objective was to carry out a brief review of the literature on $\mathrm{FBC}$ and to understand its influence on the organism, whether or not it is linked to the practice of physical activity (PA). The present study was based on a narrative review on the $\mathrm{FBC}$ theme. As conclusion, the BC is able to influence the functioning of the human body and can now be analyzed in five levels. The more sophisticated its quantification, the better its understanding of its relation to the variation of the organism's health condition (nutritional state, metabolism and pathologies). Exercise can benefit the $\mathrm{BC}$ by maintaining and increasing the components that positively influence health.

KEYWORDS: Body Composition; Movement; Health. 


\section{INTRODUÇÃO}

O estudo da composição corporal humana envolve a quantificação "in vivo" dos componentes corporais (músculos, ossos, gordura, órgãos, sistemas, etc.) e suas relações associadas a fatores como nutrição, exercício físico, crescimento, desenvolvimento, envelhecimento e doenças associadas (SARDINHA, 2012). Nessa direção, se estabelece o conceito de Composição Corporal Funcional (CCF), um ramo da biologia humana que integra os componentes corporais em sistemas de regulação (por exemplo, no equilíbrio energético), considerando suas relações quanti-qualitativamente.

Como exemplo, a regulação do peso corporal (PC) está intimamente ligada à massa e à função de vários componentes isoladamente. A perspectiva da CCF contribui para uma visão mais sofisticada sobre os efeitos e significados dos elementos envolvidos na estimativa desse PC. O controle ponderal então é observado num contexto de estado nutricional e doenças, bem como a partir da caracterização de traços biomédicos que irão fornecer evidências e direcionamentos a intervenções mais eficazes. Quantificar os componentes corporais e as alterações decorrentes de fatores como exercício físico, nutrição, crescimento, doenças e envelhecimento é essencial para a qualidade de vida e de saúde dos indivíduos, bem como para os profissionais que atuam na área do exercício e da saúde (SARDINHA, 2012). Assim, um conhecimento aprofundado acerca da Composição Corporal (CC) do indivíduo pode ser importante para a prevenção e o controle de várias doenças (obesidade, diabetes, doenças cardiovasculares, dentre outras) (CASAZZA et al., 2013; HELLER, 2009; HEYMSFIELD, et al., 2014; MULLER; BOSY-WESTPHAL).

Desta forma, a perspectiva da CCF considera uma interpretação mais incisiva das funções do organismo e seus distúrbios a partir dos componentes do próprio organismo (MULLER, 2013), compreendendo um entendimento mais sofisticado do estado nutricional, metabólico/endócrino e de doenças do indivíduo para além de apresentar evidências funcionais relativas a variantes genéticas (MULLER et al., 2009).

Dentre as possibilidades de intervenção, a atividade física (AF) é um recurso que, quando cuidadosamente alinhada aos pressupostos da CCF, pode ser um fator primordial no equilíbrio de respostas funcionais, potencializando as chances de promover um significativo impacto no estilo de vida. Isso é o que ocorre quando um programa de AF para emagrecimento estruturado é utilizado para evitar que as perdas em grande parte sejam da massa isenta de gordura (MIG). Nesse sentido, para além dos benefícios da redução do peso a AF está alinhada aos preceitos da CCF, que considera as respostas metabólicas e funcionais interpretadas para muito além das variações ponderais verificadas na balança (DONNELLY et al., 2009). Diante do exposto, o presente artigo apresenta uma breve revisão da literatura acerca dos pressupostos que envolvem a CCF e sua articulação com a prática de AF.

\section{MÉTODOS}

Trata-se de um estudo de revisão da literatura. As bases de dados utilizadas foram Pubmed e Scielo. O período de publicação dos artigos selecionados foi de 1990 a 2014. Os descritores utilizados foram composição corporal funcional e componentes corporais. Foram incluídos: a) estudos clínicos com delineamento transversal, longitudinal, de caso controle e aleatorizados, além de estudos de revisão relacionados à CCF; b) estudos publicados no idioma inglês (tanto as bases de dados como o idioma foram definidos com base no objeto de estudo). Foram excluídos artigos com informações incompletas que não atenderam aos critérios de elegibilidade.

Inicialmente os artigos foram eleitos de acordo com os critérios descritos acima. Esta revisão compreendeu três fases. Na primeira foi realizada a pesquisa nos bancos de dados Pubmed e Scielo. Na segunda fase após a seleção foram realizadas análises dos títulos e resumos até o ano de 2014 para determinar a elegibilidade dos estudos. Na terceira e última fase foram avaliados os textos completos. Por fim, foi organizado um fluxograma com o propósito de sistematização (Figura 1). Foram realizadas análises críticas de conteúdo, considerando o mérito científico de cada estudo e possíveis relações de similaridades ou conflitos entre eles. Este processo foi realizado por dois pesquisadores de forma independente. 


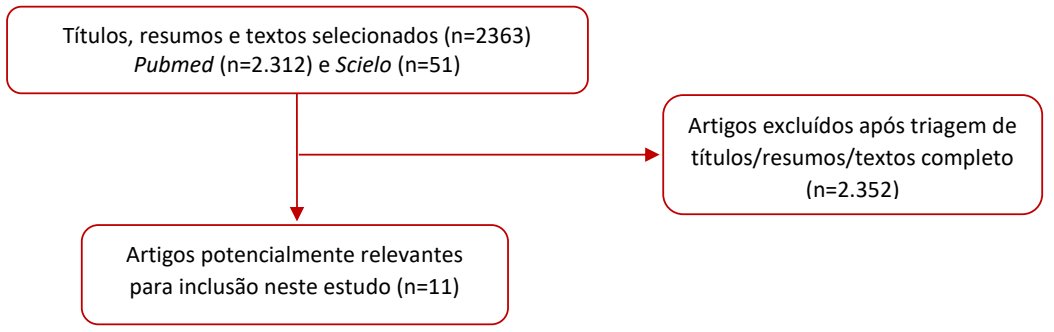

Figura 1. Fluxograma da seleção dos artigos científicos.

\section{RESULTADOS E DISCUSSÃO}

Após consulta às bases de dados foram encontradas 2.363 publicações sobre o assunto: Pubmed $(n=2.312)$ e Scielo $(n=51)$. Após a triagem de títulos/resumos/texto completo foram excluídos 2.352 artigos. Apenas 11 estudos (representados no Quadro 1) foram incluídos na presente análise após a filtragem da busca, conforme critérios de seleção.

Quadro 1. Artigos utilizados para construção desta revisão narrativa.

\begin{tabular}{|c|c|c|c|}
\hline Autores & Ano & Título & Conteúdo \\
\hline Wang et al. & 1992 & $\begin{array}{l}\text { Modelos e métodos de composição } \\
\text { corporal (níveis compartimentais: } \\
\text { atômicos, moleculares, celulares, } \\
\text { tecidular e de corpo total). }\end{array}$ & $\begin{array}{l}\text { Apresenta descrições dos níveis de composição corporal } \\
\text { e suas regras de organização; I. atômico: II. molecular: III. } \\
\text { celular; IV. tecidular, e V. corpo total. }\end{array}$ \\
\hline Heymsfield et al. & 2005 & $\begin{array}{l}\text { Estamos nos aproximando do fim da } \\
\text { pesquisa sobre composição corporal? }\end{array}$ & $\begin{array}{l}\text { Os principais componentes são mensuráveis in vivo. } \\
\text { A tomografia e a ressonância magnética ampliam as } \\
\text { estimativas de "massa" para a "função" dos componentes. } \\
\text { Entretanto, algumas complexidades permanecem como } \\
\text { medir sujeitos muito jovens, sujeitos no útero e com } \\
\text { obesidade severa. Embora os principais componentes } \\
\text { possam ser mensuráveis, algumas lacunas ao considerar } \\
\text { exposição à radiação, invasividade, medidas estáticas } \\
\text { versus dinâmica e laboratorial versus clínica e avaliações } \\
\text { de campo, permanecem. }\end{array}$ \\
\hline Muller et al. & 2009 & $\begin{array}{l}\text { Composição corporal "funcional" } \\
\text { diferenciação entre obesidade benigna } \\
\text { e não - benigna. }\end{array}$ & $\begin{array}{l}\text { Recentes análises da CC detalhada, junto com avaliações } \\
\text { de resistência à insulina, aptidão aeróbia, mostrou que } \\
\text { sujeitos com obesidade benigna apresentam similares } \\
\text { índice de massa corporal (IMC), circunferência de quadril, } \\
\text { gordura abdominal subcutânea quando comparados a } \\
\text { em sujeitos com obesidade não benigna. Nesse sentido, } \\
\text { a avaliação da CCF nos proporciona uma visão mais } \\
\text { sofisticada sobre o estado nutricional, metabolismo, } \\
\text { endocrinologia e doenças. }\end{array}$ \\
\hline Muller et al. & 2009 & $\begin{array}{l}\text { Composição corporal funcional: } \\
\text { insights sobre a regulação do } \\
\text { metabolismo energético e algumas } \\
\text { aplicações clínicas. }\end{array}$ & $\begin{array}{l}\text { A CCF integra os componentes corporais em uma } \\
\text { perspectiva mais ampla do sistema regulatório. A } \\
\text { regulação do peso corporal apresenta forte associação } \\
\text { com a massa e a função dos componentes corporais. }\end{array}$ \\
\hline Donnelly et al. & 2009 & $\begin{array}{l}\text { Estratégias de intervenção de atividade } \\
\text { física adequados para a perda de peso } \\
\text { corporal e prevenção da "re-ganho" do } \\
\text { peso corporal em adultos. }\end{array}$ & $\begin{array}{l}\text { AF moderada de } 150 \text { a } 250 \text { minutos por semana, com } \\
\text { uma energia equivalente a } 1200 \text { a } 2000 \text { kcal parece ser } \\
\text { suficiente para prevenir o ganho de peso corporal na } \\
\text { maioria dos adultos e podem resultar em uma modesta } \\
\text { perda de peso. }\end{array}$ \\
\hline Ackland et al. & 2012 & $\begin{array}{l}\text { Estado atual da composição corporal e } \\
\text { avaliação no Esporte. }\end{array}$ & $\begin{array}{l}\text { Todos os métodos e procedimentos apresentam } \\
\text { problemas; seja na metodologia, interpretação de dados } \\
\text { ou mesmos nos pressupostos levados em consideração. } \\
\text { As limitaçães vão desde o modelo } 2 \mathrm{C} \text { (precisão) até nos } \\
\text { modelos multicompartimentais (praticidade). Na ausência } \\
\text { de uma técnica precisa e prática, existe uma margem de } \\
\text { erro para que os outros métodos desempenhem um papel } \\
\text { preciso. }\end{array}$ \\
\hline
\end{tabular}


Quadro 1. Artigos utilizados para construção desta revisão narrativa (continuação).

\begin{tabular}{|c|c|c|c|}
\hline Autores & Ano & Título & Conteúdo \\
\hline Casazza et al. & 2013 & $\begin{array}{l}\text { Mitos, presunções e fatos sobre } \\
\text { Obesidade. }\end{array}$ & $\begin{array}{l}\text { O artigo analisou algumas crenças comuns sobre a } \\
\text { obesidade. Essas falsas crenças e sem embasamento } \\
\text { científico estão presentes tanto na literatura científica } \\
\text { quanto na imprensa popular }\end{array}$ \\
\hline Muller & 2013 & $\begin{array}{l}\text { Do IMC à composição corporal } \\
\text { funcional. }\end{array}$ & $\begin{array}{l}\text { CCF mostra efeitos das doenças na composição do } \\
\text { organismo e sua interação com estes, para além de uma } \\
\text { estrita inter-relação. }\end{array}$ \\
\hline Heymsfield et al. & 2014 & $\begin{array}{l}\text { Avaliando massa muscular esquelética: } \\
\text { panorama histórico e estado da arte. }\end{array}$ & $\begin{array}{l}\text { A partir da observação da carne como fonte primária de } \\
\text { creatina, emergiram novos métodos para medir a massa } \\
\text { do músculo esquelético humano e sua composição. }\end{array}$ \\
\hline Heymsfield et al. & 2014 & $\begin{array}{l}\text { Composição da perda de peso corporal } \\
\text { é equivalente a um quarto de massa } \\
\text { isenta de gordura (MlG): uma revisão } \\
\text { crítica desta regra amplamente citada. }\end{array}$ & $\begin{array}{l}\text { Regra } 1 / 4 \text { MIG é na melhor das hipóteses uma aproximação } \\
\text { que tem uma base limitada, pois a perda de MIG varia ao } \\
\text { longo do tempo e é determinada por vários fatores. }\end{array}$ \\
\hline Muller et al. & 2014 & $\begin{array}{l}\text { CCF e aspectos relacionados em } \\
\text { pesquisa sobre obesidade e caquexia: } \\
\text { relatório sobre a } 12^{a} \text { da Conferência } \\
\text { realizada em } 6 \text { e } 7 \text { de Setembro de } \\
\text { 2013, em Hamburgo, Alemanha. }\end{array}$ & $\begin{array}{l}\text { O estudo apresenta uma integração de ideias e } \\
\text { investigação sobre as semelhanças e as condições } \\
\text { extremas de obesidade e caquexia. }\end{array}$ \\
\hline
\end{tabular}

\section{CONTEXTUALIZANDO A COMPOSIÇÃO CORPORAL FUNCIONAL}

De um ponto de vista histórico, a análise da CC teve o primeiro modelo de dois compartimentos (2C) proposto por Behnke (1942) para estimar massa gorda (MG) e MIG, mesmo que apresentassem densidades específicas de 0,9007 g/mL e 1,100 g/mL, respectivamente (BROZEK et al., 1963). Apenas essa diferença já limitava a interpretação das alterações biológicas ocorridas no corpo humano, pois considerava uma densidade única, estimada a partir de técnicas densitométricas.

Posteriormente, Siri incluiu a estimativa de um terceiro componente, a água corporal total, originando o modelo a três compartimentos (3C) (SIRI, 1961). Mais tarde, o modelo multicompartimental usando métodos ou combinações de métodos para medir a CC que considerava três ou mais componentes da MIG também foi desenvolvido. Assim, a avaliação da CC se tornava mais acurada de acordo com o número de componentes medidos (WELLS et al., 1999). O modelo de quatro componentes (4C), por exemplo, considera valores específicos de densidade para gordura $(0,9007 \mathrm{~g} / \mathrm{mL})$, água $(0,9937 \mathrm{~g} / \mathrm{mL})$, mineral $(3,038 \mathrm{~g} / \mathrm{mL})$ e proteína $(1,34 \mathrm{~g} / \mathrm{mL})$ (BROZEK et al., 1963).

O estudo da CC apresenta três áreas interconectadas: 1) os níveis de CC e suas regras de organização, 2) a medição técnica e 3) os fatores biológicos que a influenciam. A primeira área estabelece os conceitos da CC e a organiza de acordo com cinco níveis de elementos corporais: atômico, molecular, celular, órgãotecidual e corpo total (WANG; PIERSON; HEYMSFIELD, 1992). O modelo de cinco níveis, proposto por Wang e colaboradores, apresenta uma estrutura adequada para a investigação dos componentes corporais e suas relações com alterações biológicas (HEYMSFIELD et al., 2014; WANG; PIERSON; HEYMSFIELD, 1992), conforme detalhado mais adiante.

A segunda área envolve a medição técnica da CC, voltada para o estudo e a aplicação de métodos de quantificação dos componentes corporais nos diferentes níveis, seja atômico (I), molecular (II), celular (III), órgão-tecidual (IV) ou de corpo total (V) (HEYMSFIELD et al., 2005). Diferentes métodos estão disponíveis para medidas "in vivo" e "in vitro" dentro dos principais componentes dos cinco níveis apresentados. Muitos métodos permitem estabelecer relações intra-sujeitos dentre os componentes intra e inter níveis, permitindo formulação de modelos específicos ou novas formas de combinações (MACHADO, 2009).

A terceira questão está relacionada a sua variabilidade biológica intra e inter sujeitos que envolvem as mudanças na CC relacionada às condições individuais fisiológicas e patológicas. As considerações desta questão nas investigações incluem fatores de crescimento, desenvolvimento, envelhecimento, raça, nutrição, efeitos hormonais e AF, bem como algumas doenças e medicamentos que podem afetar a CC (MACHADO, 2009). 
A CCF integra os componentes do corpo em sistemas regulatórios, isto é, relaciona a CC com sua função in vivo e processos metabólicos correspondentes. As aplicações adequadas e a análise da CC são (i) a interpretação das funções do corpo (por exemplo, gasto de energia, sensibilidade à insulina) e seus distúrbios no contexto dos componentes do corpo e vice-versa e (ii) a interpretação do significado de componentes corporais individuais no contexto de suas consequências funcionais. Ou seja, como diferentes funções corporais e processos metabólicos estão relacionados de forma diversa aos componentes corporais individuais, a CCF amplia a visão tradicional, cruzando e combinando os diferentes níveis da CC, proporcionando uma visão mais sofisticada e detalhada sobre o estado nutricional, o metabolismo endócrino e as doenças (MULLER et al., 2009; MULLER, 2013).

\section{ENTENDENDO OS COMPONENTES CORPORAIS}

Para analisar e compreender a CC de maneira efetiva o modelo mais aceito na área é o proposto por Wang e colaboradores (WANG; PIERSON; HEYMSFIELD, 1992), que expressa os diferentes níveis de complexidade do corpo humano organizados em cinco níveis: atômico, molecular, celular, órgão-tecidual e de corpo total. Certos princípios são inerentes a este modelo e ultimamente as propostas de CC tendem a obedecer a esta conceituação. Os compartimentos são distintos e estão conectados pelas reações bioquímicas e fisiológicas do organismo. A soma de todos os componentes em cada nível é equivalente à massa corporal total (Figura 2).

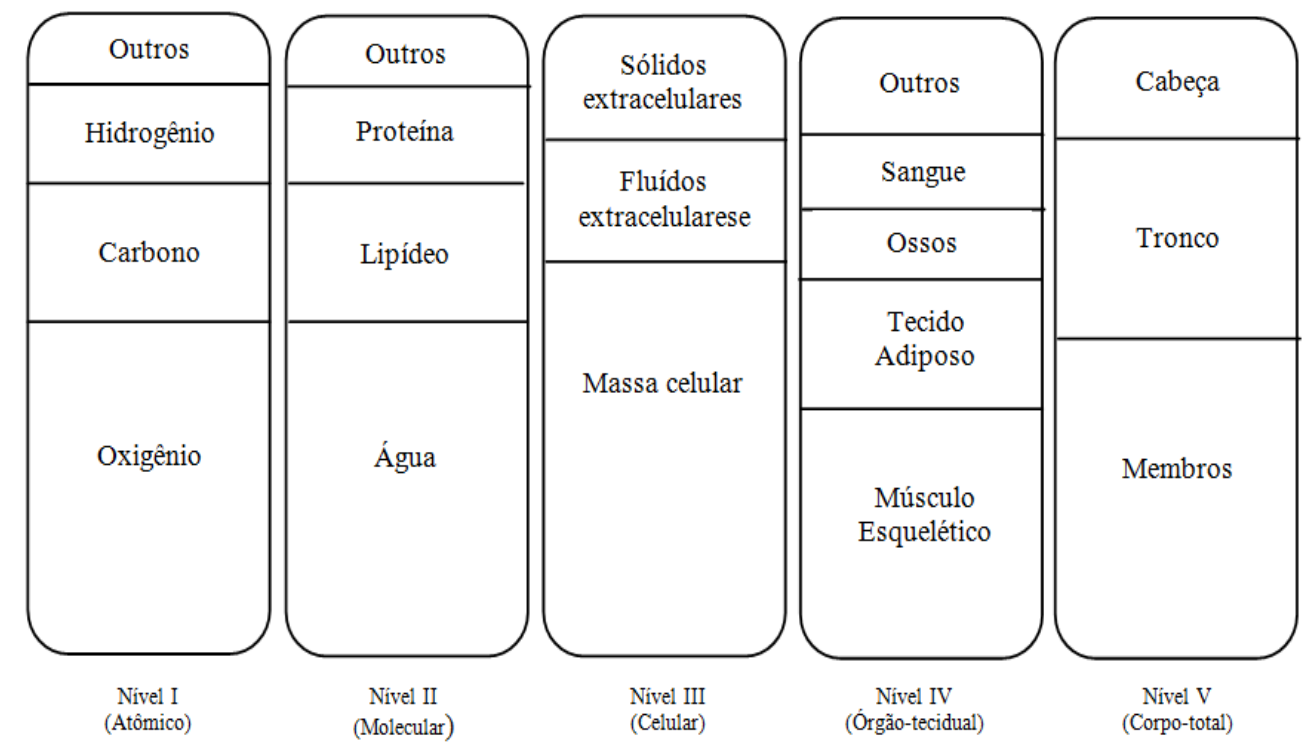

Figura 2. Modelo dos cinco níveis da composição corporal. Adaptado de WANG et al. (1992).

As investigações nessa área podem se iniciar por meio de análises mais elementares com a quantificação bi-compartimental da CC, mas a análise de mais compartimentos pode fornecer informações mais precisas da relação entre o organismo e a CC em cada nível (ELLIS, 2000). Vale destacar que cada nível é constituído de vários elementos e uma análise em nível permite uma visão detalhada do corpo humano. Assim, é possível quantificar os elementos básicos de interesse (ie. o nível atômico possui cerca de 50 elementos, sendo que o oxigênio, carbono, hidrogênio, nitrogênio, cálcio e fósforo são responsáveis por $98 \%$ da massa corporal. (MULLER et al., 2009; WANG; PIERSON; HEYMSFIELD, 1992).

Entretanto, os elementos do organismo sofrem influência endógena e exógena constantemente e o termo estado estável da CC, proposto por Wang e colaboradores, assinala que existe um equilíbrio dinâmico do organismo desde que as relações entre as massas de vários componentes em diferentes níveis sejam mantidas 
relativamente constantes, mesmo durante o ganho ou perda de PC (MULLER et al., 2014). Por exemplo, no nível molecular, a razão entre a quantidade total de água corporal e MIG em um indivíduo saudável é igual a 0,732; qualquer alteração dessas relações indica um mau funcionamento do organismo e a necessidade de uma investigação mais aprofundada.

Para explicar como os tipos de influência agem sobre o equilíbrio dinâmico do organismo, a CCF também utiliza os termos massa dependente e massa independente da CC. Sendo assim, certas respostas do organismo ocorrem devido à alteração de determinados componentes, como o aumento da MIG (massa dependente) está relacionado com o aumento do gasto energético de repouso e a obesidade está associada com altos níveis de leptina no plasma (também massa dependente). Outras respostas do organismo ocorrem devido a determinadas situações externas, como jejum, excesso de alimentação, estresse, doenças, etc. (massa independente), que podem proporcionar aumento ou diminuição do PC (MULLER et al., 2009). A análise no nível molecular proposto por Wang et al. (1992) possibilita avaliar vários componentes, como ilustra a Figura 3.

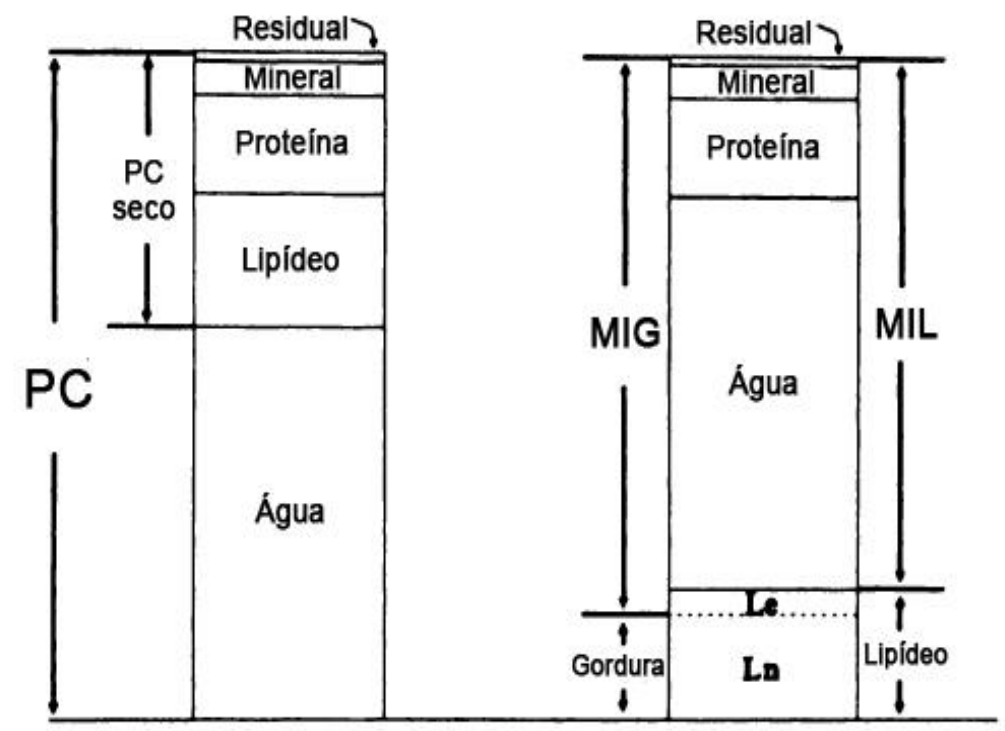

Figura 3. Modelo de composição corporal no nível molecular adaptado de Wang et al. (1992). Legenda: definidos por Peso Corporal (PC; seco + úmido) e Massa Isenta de Gordura (MIG; MIL: Massa Isenta de Lipídeo) + Gordura (Le: Lipídeo essencial; Ln: Lipídeo não essencial).

Conforme retratado, a água é o composto químico mais abundante no corpo do ser humano, compreendendo $70 \%$ do PC total; a Proteína tem como aliados quase todos os compostos que contêm nitrogênio, variando a partir de aminoácidos simples a complexos; o Mineral é uma categoria de inorgânico que contêm grande quantidade de elementos de metal (cálcio, sódio e potássio) e elementos metaloides (oxigênio, fósforo, e cloro) e os Minerais são normalmente divididos em duas subcategorias: ósseo e extra ósseo.

Por fim, os lipídeos e as gorduras são utilizados como sinônimos corriqueiramente. Entretanto Wang et al. (1992) destacam que as gorduras são parte constituinte dos lipídeos. As gorduras são sinônimas de lipídeo não essenciais (Ln), representando cerca de $90 \%$ do conteúdo total de lipídeos. Os lipídeos, além de serem constituídos pelas gorduras ou Ln, ainda contam com a participação dos lipídeos essenciais (Le), contabilizando aproximadamente $10 \%$ do conteúdo total de lipídeos.

Os Le, tais como esfingomielina e fosfolipídeos, exercem funções importantes, como a formação das membranas celulares e os Ln, em grande parte sob a forma de triglicérides, podem proporcionar isolamento térmico e atuar como uma reserva energética (WANG; PIERSON; HEYMSFIELD, 1992). 


\section{DISTÚRBIO DA COMPOSIÇÃO CORPORAL}

A obesidade e o sobrepeso respondem por dimensionar o acúmulo de gordura corporal, normalmente associado a vários distúrbios funcionais, metabólicos e comorbidades. Contribuem com muitos fatores para o desenvolvimento de múltiplas doenças como cardiopatia, hipertensão, diabetes e alguns tipos de câncer (DONNELLY et al., 2009). O risco de doenças cardiometabólicas pode variar de acordo com a distribuição de gordura corporal ou padrão de gordura. Atualmente, os padrões de gordura se dividem como androide, apresentando maior concentração de gordura no tronco e menor quantidade nas extremidades, e ginoide, com maior quantidade de gordura na região dos quadris e extremidades e redução de gordura no tronco (VAGUE, 1947). A gordura visceral, localizada no tronco, é considerada mais metabolicamente ativa do que a gordura subcutânea e um fator de risco para doenças cardiovasculares, resistência à insulina e diabetes mellitus tipo 2 (DESPRES et al., 2008). Por outro lado, menor quantidade de gordura subcutânea nas extremidades está associada ao aumento da sensibilidade à insulina e pode ser protetora contra o desenvolvimento de doenças cardiometabólicas através da supressão dos ácidos graxos livres (AMATI et al., 2012).

Nesse sentido, o aumento da quantidade de MG geral e o acúmulo de gordura abdominal estão associados com distúrbios metabólicos e comorbidades (MULLER, 2013). Dentre essas doenças, destaca-se a obesidade, que pode ocorrer por fatores externos e/ou biológicos. Atualmente, esta doença afeta grande parte da população infantil e adulta. Em seu estudo, Donnelly et al. (2009) observaram que a diminuição do PC traz benefícios para a saúde, minimizando a chance do indivíduo desenvolver algum tipo de doença crônica. Entretanto, o acúmulo ou a distribuição de gordura de forma isolada não são fatores preditivos de estados patológicos, pois 30\% dos obesos são considerados metabolicamente saudáveis (MULLER, 2013).

\section{SARCOPENIA}

O envelhecimento está associado a deficiências metabólicas, fisiológicas e funcionais, em parte por mudanças relacionadas à idade na CC. O PC geralmente aumenta até 80 anos, porém, apresenta um declínio após essa idade. Mesmo quando o PC permanece estável, as alterações na composição dos tecidos podem ser detectadas. Análises detalhadas mostraram que além de uma redução da massa muscular (MM) ocorre também um aumento da infiltração de gordura nos músculos. Embora a gordura corporal total diminua em idades muito avançadas, a infiltração de gordura nos músculos parece continuar com as mudanças relativas entre 15 e 30\% (MULLER et al., 2014).

Mudanças na MM relacionadas à idade impactam sobre a sua função, sendo a perda desse compartimento considerada um dos principais determinantes da redução de força no envelhecimento. Um declínio anual de $1 \%$ da MM com o envelhecimento é acompanhado por uma queda de $3 \%$ na força muscular. Assim, a diminuição de força é muito mais acelerada do que a concomitante perda de MM (MULLER et al., 2014).

Esse conjunto de alterações é definido como sarcopenia, uma doença (ANKER; MORLEY; HAEHLING, 2016) caracterizada pela redução da massa muscular e força muscular (CRUZ-JENTOFT et al., 2010), muitas vezes associada somente ao envelhecimento (DONNELLY et al., 2009; MULLER; BOSY-WESTPHAL; HELLER, 2009) ou resultante de outros fatores claramente perceptíveis, como, por exemplo, desnutrição, patologias que afetam o sistema de desequilíbrio energético, problemas endócrinos e doenças neurodegenerativas. Essa condição pode influenciar diretamente na funcionalidade de indivíduos idosos, uma vez que triplica o risco de quedas, influencia na perda da independência motora, além de elevar o risco de morte prematura (KIM et al., 2014; LAURETANI et al., 2003; WU et al., 2014).

A mensuração dos componentes corporais, como por exemplo, a massa isenta de gordura e osso apendicular é primordial para identificar a sarcopenia. Esse distúrbio da CC deve ser prevenido ou combatido, visto que suas consequências impactam diretamente em aspectos da economia e da saúde pública (BEAUDART et al., 2014). Recentemente estudos retrataram uma prevalência da doença de $17 \%$ no Brasil (DIZ et al., 2017), número que tende a elevar devido ao aumento esperado de idosos em todo o mundo, passando dos 605 milhões atuais, para aproximadamente dois bilhões no ano de 2050 , sendo que $80 \%$ desses indivíduos residirão em países subdesenvolvidos, como é o caso do Brasil (WORLD HEALTH ORGANIZATION, 2014). 
Portanto, a utilização dos métodos e técnicas para mensuração da CC são fundamentais para identificação dos distúrbios da CC e assim, permitem o correto direcionamento das ações para prevenir/combater esses distúrbios.

\section{MÉTODOS E TÉCNICAS DE APLICAÇÃO PARA ANÁLISE DA COMPOSIÇÃO CORPORAL}

Os componentes do organismo, quando avaliados de maneira individualizada por diferentes métodos e técnicas, ajudam na compreensão dos detalhes da CC nos diferentes níveis (atômico, molecular, celular, órgão-tecidual e corpo total). Assim, é possível identificar tipos específicos de desnutrição através da gordura corporal e da massa muscular, como, por exemplo, a identificação da sarcopenia ou outros distúrbios (MULLER; BOSY-WESTPHAL; HELLER, 2009). Sendo assim, é necessário entender quais são os métodos e técnicas que podem ser aplicados para análise da CCF. Alguns métodos são reportados na literatura (ACKLAND et al., 2012) da estimativa da CC, conforme explicitados no Quadro 2.

Quadro 2. Métodos e técnicas utilizados para análise da composição corporal.

\begin{tabular}{|c|c|c|}
\hline & \multicolumn{2}{|c|}{ Método Direto } \\
\hline & Dissecação de cadáver & Densitometria \\
\hline Físico-Químico & Métodos Indiretos & Pesagem Hidrostática \\
Pletismografia & Imagem & Hidrometria \\
Absorção de gases & Raios-x & \\
Diluição de isótopos & Ressonância magnética \\
Espectrometria de raios gama & Densitometria de dupla energia (DXA) \\
\hline Espectrofotometria & Ultrassom & \\
Excreção de creatinina & Tri-dimensional (3D) & \\
\hline & Duplamente indiretos & \\
\hline
\end{tabular}

A dissecação de cadáver é o único método considerado como direto na análise da CC. Portanto, para determinação da validade de qualquer outro método e suas medidas estes devem ser prioritariamente comparados com esta abordagem. No entanto, esse método também tem várias limitações, incluindo questões éticas, e não deve ser utilizado para análise individual (ACKLAND et al., 2012). A seguir, serão abordados alguns outros métodos, como os indiretos e os duplamente indiretos, de acordo com seus respectivos níveis de análise.

O método da análise do potássio corporal total compreende o nível I (atômico) da CC (MULLER et al., 2009) e tem como objetivo estimar a MIG por meio da contagem de potássio existente no corpo, através da técnica de espectrometria de raios gama. O método é invasivo, de alto custo e necessita de profissionais muito qualificados, sendo utilizado somente em laboratórios especializados (MONTEIRO; FILHO, 2002).

A densitometria de dupla energia (DXA) é uma técnica de imagem no nível II (molecular) da CC que foi desenvolvida, a princípio, para medir o conteúdo mineral ósseo (CMO) e a densidade mineral óssea por região e de corpo total. Contudo, o desenvolvimento da análise de corpo inteiro, através de raios-x com fótons de dupla energia, permite também quantificar a MG e MIG (LUKASKI, 1993). Esse instrumento avalia a CC de forma multicompartimentada (MG, MIG e CMO) e representa o maior avanço no laboratório e na prática clínica para estimar a CC. Os métodos duplamente indiretos, em específico os de campo, são frequentemente utilizados para monitorar a CC tanto no campo da saúde como no esportivo, mas com diferentes graus de validade (ACKLAND et al., 2012). 
Os métodos por imagem, como ressonância magnética e tomografia computadorizada, também são utilizados para análise da CC no nível IV (órgão-tecidual) (MULLER et al., 2009). A ressonância magnética utiliza um potente campo magnético e ondas de rádio frequência para produzir imagens detalhadas dos órgãos e tecidos. Enquanto a tomografia computadorizada recolhe informações após expor o corpo a uma sucessão de raios-x. Os dois métodos possuem suas medidas válidas quando comparados à dissecação de cadáveres (MITSIOPOULOS et al., 1998) e são considerados precisos, no entanto de alto custo, além de serem de difícil acesso (QUITERIO et al., 2009). Especificamente a tomografia expõe os avaliados à radiação, o que limita a utilização desses instrumentos de forma contínua (LEE et al., 2000).

Outro método frequentemente utilizado para analisar a CC é a bioimpedância elétrica (BIA), que compreende o nível III (celular) e geralmente é utilizada em estudos populacionais. O exame acontece por meio da fixação de eletrodos na pele que transmitem uma corrente elétrica que percorre o corpo de modo inofensivo, sendo possível medir a resistência à passagem dessa corrente e obter ainda outras medidas, dependendo do hardware da BIA. Esta medida permite estimar o percentual de gordura corporal, além de outros parâmetros, como a água corporal total, a MIG e o ângulo de fase. Assim, o método é considerado não invasivo, portátil, de aplicação rápida, com custo relativamente baixo e apresenta variações mínimas intra e interavaliador, desde que os pré-requisitos para o exame, principalmente relativos à hidratação, sejam abordados (NEVES et al., 2015).

O método mais frequentemente utilizado para identificar o estado nutricional (obesidade, sobrepeso ou baixo PC) é a antropometria, por meio do índice de massa corporal (IMC), determinado pela razão massa corporal $(\mathrm{kg})$ e estatura ao quadrado $\left(\mathrm{m}^{2}\right)$. Embora tenha validade para indicação epidemiológica, no uso individual é muito limitado na distinção pondero-estatural, caso os valores do PC estejam associados prioritariamente ao componente muscular ou adiposo. Para complementar as informações de adiposidade é possível utilizar a medida de circunferência da cintura $(\mathrm{cm})$, que permite predizer riscos cardiovasculares associados à quantidade de gordura nesta região (MULLER et al., 2009; MULLER; BOSY-WESTPHAL; HELLER, 2009). No entanto, esse método não diferencia tecido adiposo visceral e subcutâneo (TURCATO et al., 2000). Essas medidas são consideradas pertencentes ao nível mais rudimentar da CC, ou seja, o nível V (corpo inteiro).

Atualmente existem vários métodos e técnicas para medida da CC, porém os mais precisos são realizados em laboratórios e têm alto custo, fator que dificulta a aplicação em grandes populações. Por outro lado, os métodos duplamente indiretos geralmente são práticos e utilizados em pesquisa de campo por serem de baixo custo, porém é necessário ter uma atenção especial ao cálculo do erro técnico de medida, intra e interavaliador para que as medidas possam ser consideradas com exatidão.

\section{ATIVIDADE FÍSICA: UM BENEFÍCIO PARA A SAÚDE}

Mesmo quando o indivíduo não apresente redução do PC existem evidências sobre a influência da prática de AF na melhora da saúde. Tanto as atividades aeróbias quanto as anaeróbias auxiliam na prevenção de algumas doenças metabólicas, como diabetes tipo 2, hipertensão e doenças cardíacas (DONNELLY et al., 2009). AF é recomendada como um componente de gerenciamento do PC seja para seu aumento ou redução. Em uma pesquisa publicada pelo American College of Sports Medicine (ACSM) em 2001 (DONNELLY et al., 2009) foi recomendado aos adultos com sobrepeso ou obesos um mínimo de 150 min de AF moderada por semana para adquirir hábitos saudáveis, buscando melhorar suas condições de saúde. Da mesma forma, prática de AF de 200-300 min por semana foi recomendada para a redução de PC a longo prazo (DONNELLY et al., 2009).

Na literatura, há muito tempo se propaga que, em programas para redução de PC, $25 \%$ do peso perdido é na forma de $M M$, principalmente, quando são utilizadas somente dietas hipocalóricas (6). Entretanto, é importante destacar que 1) o emagrecimento nem sempre está associado à redução do PC, pois essa ocorre apenas quando há redução de gordura e 2) a perda de MM varia ao longo do tempo, sendo determinada por vários fatores, mesmo frente à prática de exercício físico. O exercício, por exemplo, é visto como uma forma de poupar a MM, mesmo que pequenas mudanças ponderais, quando corretamente administradas, refletem perdas de MG e ganhos de MM. Mesmo não ocorrendo aumentos relevantes de MM, em programas 
de redução do PC, o exercício físico é importante para a manutenção desse compartimento tão importante para as atividades da vida diária (HEYMSFIELD et al., 2014).

\section{CONSIDERAÇÕES FINAIS}

Por meio da CCF é possível compreender de forma mais incisiva as funções do organismo humano a partir dos componentes do próprio organismo, tanto em seus distúrbios como na sua normalidade. Isso proporciona um entendimento sofisticado do estado nutricional, metabólico/endócrino e de doenças do indivíduo, além de apresentar evidências funcionais relativas a variantes genéticas.

O modelo dos cinco níveis proposto por Wang e colaboradores é reconhecido na literatura como um padrão organizacional aceitável, aplicado para compreender e aprofundar o conhecimento da CCF. Métodos e técnicas de medida da CC apresentados neste estudo servem para diagnosticar e acompanhar a CCF.

Finalmente, o estudo sugere que a análise da CCF realizada por meio de métodos e técnicas adequadas é o modo mais apropriado para os profissionais da saúde monitorarem as relações entre a CC e o funcionamento do organismo, auxiliando também a identificar distúrbios da CC permitindo o direcionamento das ações e tratamentos.

\section{REFERÊNCIAS}

ACKLAND, T. R.; LOHMAN, T. G.; SUNDGOT-BORGEN, J.; MAUGHAN, R. J.; MEYER, N. L.; STEWART, A. D.; MULLER, W. Current status of body composition assessment in sport: review and position statement on behalf of the ad hoc research working group on body composition health and performance, under the auspices of the IOC Medical Commission. Sports Medicine, Auckland, v. 42, n. 3, p. 227-46, 2012.

AMATI, F.; PENNANT, M.; AZUMA, K.; DUBE, J. J.; TOLEDO, F. G.; ROSSI, A. P.; KELLEY, D. E.; GOODPASTER, B. H. Lower thigh subcutaneous and higher visceral abdominal adipose tissue content both contribute to insulin resistance. Obesity, Silver Spring, v. 20, n. 5, p. 1115-7, 2012.

ANKER, S. D.; MORLEY, J. E.; HAEHLING, S. Welcome to the ICD-10 code for sarcopenia. Journal of Cachexia, Sarcopenia and Muscle, Berlin, v. 7, n. 5, p. 512-4, 2016.

BEAUDART, C.; RIZZOLI, R.; BRUYÈRE, O.; REGINSTER, J.-Y.; BIVER, E. Sarcopenia: burden and challenges for public health. Archives of Public Health, Brussels, v. 72, n., p. 45, 2014.

BEHNKE, A. R. Physiologic studies pertaining to deep sea diving and aviation, especially in relation to the fat content and composition of the body: the harvey lecture, march 19, 1942. Bulletin of the New York Academy of Medicine, New York, v. 18, n. 9, p. 561-85, 1942.

BROZEK, J.; GRANDE, F.; ANDERSON, J. T.; KEYS, A. Densitometric analysis of body composition: revision of some quantitative assumptions. Annals of the New York Academy of Sciences, New York, v. 110 , n. 1 , p. $113-40,1963$.

CASAZZA, K.; FONTAINE, K. R.; ASTRUP, A.; BIRCH, L. L.; BROWN, A. W.; BOHAN BROWN, M. M.; DURANT, N.; DUTTON, G.; FOSTER, E. M.; HEYMSFIELD, S. B. Myths, presumptions, and facts about obesity. New England Journal of Medicine, Boston, v. 368, n. 5, p. 446-54, 2013.

CRUZ-JENTOFT, A. J.; BAEYENS, J. P.; BAUER, J. M.; BOIRIE, Y.; CEDERHOLM, T.; LANDI, F.; MARTIN, F. C.; MICHEL, J.-P.; ROLLAND, Y.; SCHNEIDER, S. M. Sarcopenia: European consensus on definition and diagnosis report of the european working group on sarcopenia in older people. Age and Ageing, London, v. 39, n. 4, p. 412-23, 2010.

DESPRES, J. P.; ARSENAULT, B. J.; COTE, M.; CARTIER, A.; LEMIEUX, I. Abdominal obesity: the cholesterol of the 21st century? Canadian Journal of Cardiology, Oakville, v. 24, n. Suplemento d, p. 7-12, 2008.

DIZ, J. B. M.; LEOPOLDINO, A. A. O.; MOREIRA, B. D. S.; HENSCHKE, N.; DIAS, R. C.; PEREIRA, L. S. M.; OLIVEIRA, V. C. Prevalence of sarcopenia in older brazilians: a systematic review and meta-analysis. Geriatrics \& Gerontology International, Tokyo, v. 17, n. 1, p. 5-16, 2017. 
DONNELLY, J. E.; BLAIR, S.; JAKICIC, J.; MANORE, M.; RANKIN, J.; SMITH, B. American college of sports medicine position stand. Appropriate physical activity intervention strategies for weight loss and prevention of weight regain for adults. Medicine and Science in Sports and Exercise, Madison, v. 41, n. 2, p. 459-71, 2009.

ELLIS, K. J. Human body composition: in vivo methods. Physiological Reviews, Massachusetts, v. 80, n. 2, p. 649-80, 2000.

HEYMSFIELD, S. B.; ADAMEK, M.; GONZALEZ, M. C.; JIA, G.; THOMAS, D. M. Assessing skeletal muscle mass: historical overview and state of the art. Journal of Cachexia, Sarcopenia and Muscle, Berlin, v. 5, n. 1, p. 9-18, 2014.

HEYMSFIELD, S. B.; PIETROBELLI, A.; WANG, Z.; SARIS, W. H. The end of body composition methodology research? Current Opinion in Clinical Nutrition \& Metabolic Care, London, v. 8, n. 6, p. 591-4, 2005.

HEYMSFIELD, S.; GONZALEZ, M.; SHEN, W.; REDMAN, L.; THOMAS, D. Weight loss composition is onefourth fat-free mass: a critical review and critique of this widely cited rule. Obesity Reviews, Oxford, v. 15, n. 4, p. 310-21, 2014.

KIM, J. H.; LIM, S.; CHOI, S. H.; KIM, K. M.; YOON, J. W.; KIM, K. W.; LIM, J.-Y.; PARK, K. S.; JANG, H. C. Sarcopenia: An Independent Predictor of Mortality in Community-Dwelling Older Korean Men. The Journals of Gerontology Series A: Biological Sciences and Medical Sciences, Washington, v. 69, n. 10, p. 1244-52, 2014.

LAURETANI, F.; RUSSO, C. R.; BANDINELLI, S.; BARTALI, B.; CAVAZZINI, C.; DI IORIO, A.; CORSI, A. M.; RANTANEN, T.; GURALNIK, J. M.; FERRUCCI, L. Age-associated changes in skeletal muscles and their effect on mobility: an operational diagnosis of sarcopenia. Journal of Applied Physiology, Washington, v. 95, n. 5, p. 1851-60, 2003.

LEE, R. C.; WANG, Z.; HEO, M.; ROSS, R.; JANSSEN, I.; HEYMSFIELD, S. B. Total-body skeletal muscle mass: development and cross-validation of anthropometric prediction models. American Journal of Clinical Nutrition, Bethesda, v. 72, n. 3, p. 796-803, 2000.

LUKASKI, H. C. Soft tissue composition and bone mineral status: evaluation by dual-energy X-ray absorptiometry. Journal of Nutrition, Philadelphia, v. 123, n. 2, p. 438-43, 1993.

MACHADO, D. R. L. Análise multivariada da composição corporal em jovens esportistas e não esportistas. 159f. Dissertação (Doutorado em Educação Física) - Escola de Educação Física e Esporte, Universidade de São Paulo, São Paulo, 2009.

MITSIOPOULOS, N.; BAUMGARTNER, R. N.; HEYMSFIELD, S. B.; LYONS, W.; GALLAGHER, D.; ROSS, R. Cadaver validation of skeletal muscle measurement by magnetic resonance imaging and computerized tomography. Journal of Applied Physiology, Washington, v. 85, n. 1, p. 115-22, 1998.

MONTEIRO, A. B.; FERNANDES FILHO, J. Análise da composição corporal: uma revisão de métodos. Revista Brasileira de Cineantropometria e Desempenho Humano, Florianópolis, v. 4, n. 1, p. 80-92, 2002.

MULLER, M. J. From BMI to functional body composition. European Journal of Clinical Nutrition, London, v. 67, n. 11, p. 1119-21, 2013.

MULLER, M. J.; BOSY-WESTPHAL, A.; HELLER, M. Functional body composition: differentiating between benign and non-benign obesity. F1000 Biology Reports, London, v. 1, n. 1, p. 1-5, 2009.

MULLER, M. J.; BOSY-WESTPHAL; LATER, W.; HAAS, V.; HELLER, M. Functional body composition: insights into the regulation of energy metabolism and some clinical applications. European Journal of Clinical Nutrition, London, v. 63, n. 9, p. 1045-56, 2009.

MULLER, M.; BARACOS, V.; BOSY-WESTPHAL, A.; DULLOO, A.; ECKEL, J.; FEARON, K.; HALL, K.; PIETROBELLI, A.; SORENSEN, T.; SPEAKMAN, J. Functional body composition and related aspects in research on obesity and cachexia: report on the 12th stock conference held on 6 and 7 September 2013 in Hamburg, Germany. Obesity Reviews, Oxford, v. 15, n. 8, p. 640-56, 2014.

NEVES, F. S.; LEANDRO, D. A. B.; SILVA, F. A. D.; NETO, M. P.; OILEIRA, R. M. S.; CÂNDIDO, A. P. C. Evaluation of the predictive capacity of vertical segmental tetrapolar bio impedance for excess weight detection in adolescents. Jornal de Pediatria, Rio de Janeiro, v. 91, n. 3, p. 551-9, 2015. 
QUITERIO, A. L.; CARNERO, E. A.; SILVA, A. M.; BRIGHT, B. C.; SARDINHA, L. B. Anthropometric models to predict appendicular lean soft tissue in adolescent athletes. Medicine and Science in Sports and Exercise, Madison, v. 41, n. 4, p. 828-836, 2009.

SARDINHA, L. B. Functional body composition: need for a new agenda [Editorial]. Archives of Exercise, Health and Disease, Porto, v. 3, n.3, p. 183-7, 2012.

SIRI, W. E. Body composition from fluid spaces and density: analysis of method. In: BROZEK, J.; HENSCHEL, A. (Ed.). Techniques for measuring body composition. Washington: National Academy of Sciences, 1961. p. 223-44.

TURCATO, E.; BOSELLO, O.; DI FRANCESCO, V.; HARRIS, T. B.; ZOICO, E.; BISSOLI, L.; FRACASSI, E.; ZAMBONI, M. Waist circumference and abdominal sagittal diameter as surrogates of body fat distribution in the elderly: their relation with cardiovascular risk factors. International Journal of Obesity and Related Metabolic Disorders, London, v. 24, n. 8, p. 1005-10, 2000.

VAGUE, J. La differenciation sexuelle facteur determinent des formes de l'obesite. Presse Medicale, Paris, v. 55, n. 30, p. 339, 1947.

WANG, Z.-M.; PIERSON, R.; HEYMSFIELD, S. B. The five-level model: a new approach to organizing body-composition research. American Journal of Clinical Nutrition, Bethesda, v. 56, n. 1, p. 19-28, 1992.

WELLS, J. C.; FULLER, N. J.; DEWIT, O.; FEWTRELL, M. S.; ELIA, M.; COLE, T. J. Four-component model of body composition in children: density and hydration of fat-free mass and comparison with simpler models. American Journal of Clinical Nutrition, Bethesda, v. 69, n. 5, p. 904-12, 1999.

WORLD HEALTH ORGANIZATION. 10 Facts on ageing and the life course. 2014-2015. Disponível em: $<$ http://www.who.int/features/factfiles/ageing/ageing_facts/en/>. Acessado em: 10 de julho de 2017.

WU, I.; LIN, C. C.; HSIUNG, C. A.; WANG, C. Y.; WU, C. H.; CHAN, D. C. D.; LI, T. C.; LIN, W. Y.; HUANG, K. C.; CHEN, C. Y. Epidemiology of sarcopenia among community-dwelling older adults in Taiwan: A pooled analysis for a broader adoption of sarcopenia assessments. Geriatrics \& Gerontology International, Tokyo, v. 14, n. S1, p. 52-60, 2014.

\section{Autor correspondente: Anderson dos Santos Carvalho}

E-mail: ander_uai@hotmail.com

Recebido: 11 de agosto de 2017.

Aceito: 04 de abril de 2018. 